\title{
Prosthodontic guidelines for surgical reconstruction of the maxilla: A classification system of defects
}

\author{
Devin J. Okay, DDS, ${ }^{a}$ Eric Genden, MD, ${ }^{b}$ Daniel Buchbinder, DMD, MD,c and Mark Urken, MD \\ The Mount Sinai Medical Center, New York, N.Y.
}

\begin{abstract}
Surgical reconstruction of maxillectomy defects has been described as an alternative to prosthetic rehabilitation to close the oral cavity. Advancements in microvascular surgical techniques require comprehensive treatment planning guidelines for functional rehabilitation. This retrospective study evaluated acquired maxillectomy defects after surgical reconstruction and/or prosthodontic rehabilitation in an attempt to establish surgical and prosthodontic guidelines that could be organized into a classification system. Forty-seven consecutive patient treatments of palatomaxillary reconstruction at a single facility, The Mount Sinai Medical Center (New York, N.Y.), were reviewed. All patients were rehabilitated with a tissue-borne obturator, a local advancement flap, a fasciocutaneous free flap, or a vascularized bone-containing free flap. Palatomaxillary defects were divided into 3 major classes and 2 subclasses. The aim of this defect-oriented classification system was to organize and define the complex nature of the restorative decision-making process for the maxillectomy patient. (J Prosthet Dent 2001;86:352-63.)
\end{abstract}

$\mathrm{R}$ ehabilitation of the maxillectomy defect has been well defined for prosthodontists and surgeons. ${ }^{1-10}$ The primary goal of prosthetic obturation is closure of the maxillectomy defect and separation of the oral cavity from the sino-nasal cavities. A pressure-resistant seal of the obturator bulb against the mucosal lining and skin graft, if placed, restores speech and swallowing functions. A successful prosthetic design for functional restoration of the maxillectomy defect utilizes the remaining palate and dentition to maximize the support, stability, and retention of an obturator bulb (Fig. 1). An unfavorable situation for prosthetic rehabilitation occurs when the size of a defect is so large that it overwhelms the remaining structures that stabilize a prosthesis over the defect. Instability of the obturator results in air and fluid leakage through the nasal cavity and thereby compromises function. ${ }^{1-3}$

Surgical procedures for prosthetic rehabilitation help preserve and enhance supporting areas around the maxillectomy defect. ${ }^{4,5}$ These procedures include the placement of a split-thickness skin graft to improve the supporting surface of the defect and contribute to scar band formation along the lateral and posterolateral margins of the resulting cavity. Preservation of the palatal mucosa at the medial bor-

aAssistant Professor, Departments of Dentistry and OtolaryngologyHead and Neck Surgery.

bAssociate Professor, Department of Otolaryngology-Head and Neck Surgery.

cProfessor and Chair, Department of Oral and Maxillofacial Surgery.

dProfessor and Chair, Department of Otolaryngology—Head and Neck Surgery.
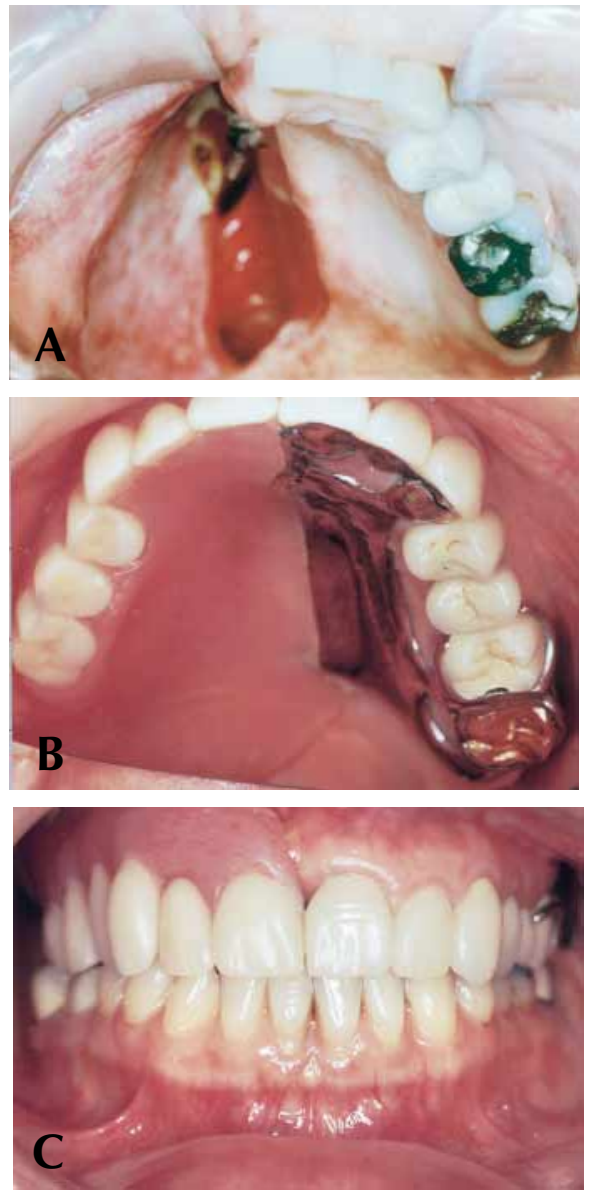

Fig. 1. A, Maxillectomy defect to be restored with definitive obturator. B, Surveyed crown restorations increased stability to obturator. C, Occlusion restored to first premolar over defect. 
der of the defect and its rotation onto the vomer improves the supporting surface within the defect as well. An osteotomy cut through an extraction site adjacent to the defect helps preserve the alveolus for the proximate abutment tooth. Coordination of surgical considerations for prosthodontic rehabilitation of maxillary defects improves the prosthetic prognosis and functional outcome.

Defect classification systems take into account the various parameters that influence the functional outcome of rehabilitation. Aramany ${ }^{6,7}$ proposed a defect classification system that presents differences of mechanics in 6 classes that are acknowledged in the prosthetic design of an obturator framework for partially edentulous patients. A definitive obturator for a partially edentulous patient has 2 main components: a metallic framework and an acrylic resin obturator bulb. The metallic framework is designed to stabilize anticipated cantilever forces along the fulcrum line defined by terminal abutment teeth. ${ }^{8-10}$ The remaining palate and the dental arch are integral to the stability of the prosthesis. Important considerations in the design of the framework are the size and location of the defect as they relate to the remaining palate and dentition. The stabilization of the obturator bulb and its intimacy with the soft tissues that line and surround the defect are thought to minimize adverse effects such as nasal leakage and hypernasal speech. Fabrication of an obturator involves fundamental principles of removable partial denture framework design and techniques that are characteristic for the maxillectomy defect.

New prosthodontic guidelines that relate to surgical reconstruction of the maxilla seem to be mandated as a result of advancements in microvascular surgical techniques. Microvascular free flap surgery allows the transfer of muscle, connective tissue, skin, and bone to recipient sites. ${ }^{11-16} \mathrm{~A}$ vascular supply to the graft can be provided after donor blood vessels are re-anastomosed to recipient vessels of the head and neck region. Fasciocutaneous and osteomyocutaneous free flaps can provide closure of the oral cavity for acquired maxillary defects. Major factors in the prosthodontic rehabilitation decisionmaking process include whether a maxillectomy defect should be reconstructed; if so, what type of free flap should be employed; and how the chosen free flap will affect the patient's oral function. In our experience, soft tissue free flaps can provide closure of the oral cavity in smaller defects but are unsupported and may not provide a stable palatal base for a removable prosthesis. If a removable prosthesis is planned with surgical closure provided by a fasciocutaneous flap, the support of the prosthesis should be derived from the remaining palate and dentition. For larger defects, the use of vascularized bone-containing free flaps (VBCFF) for maxillary reconstruction
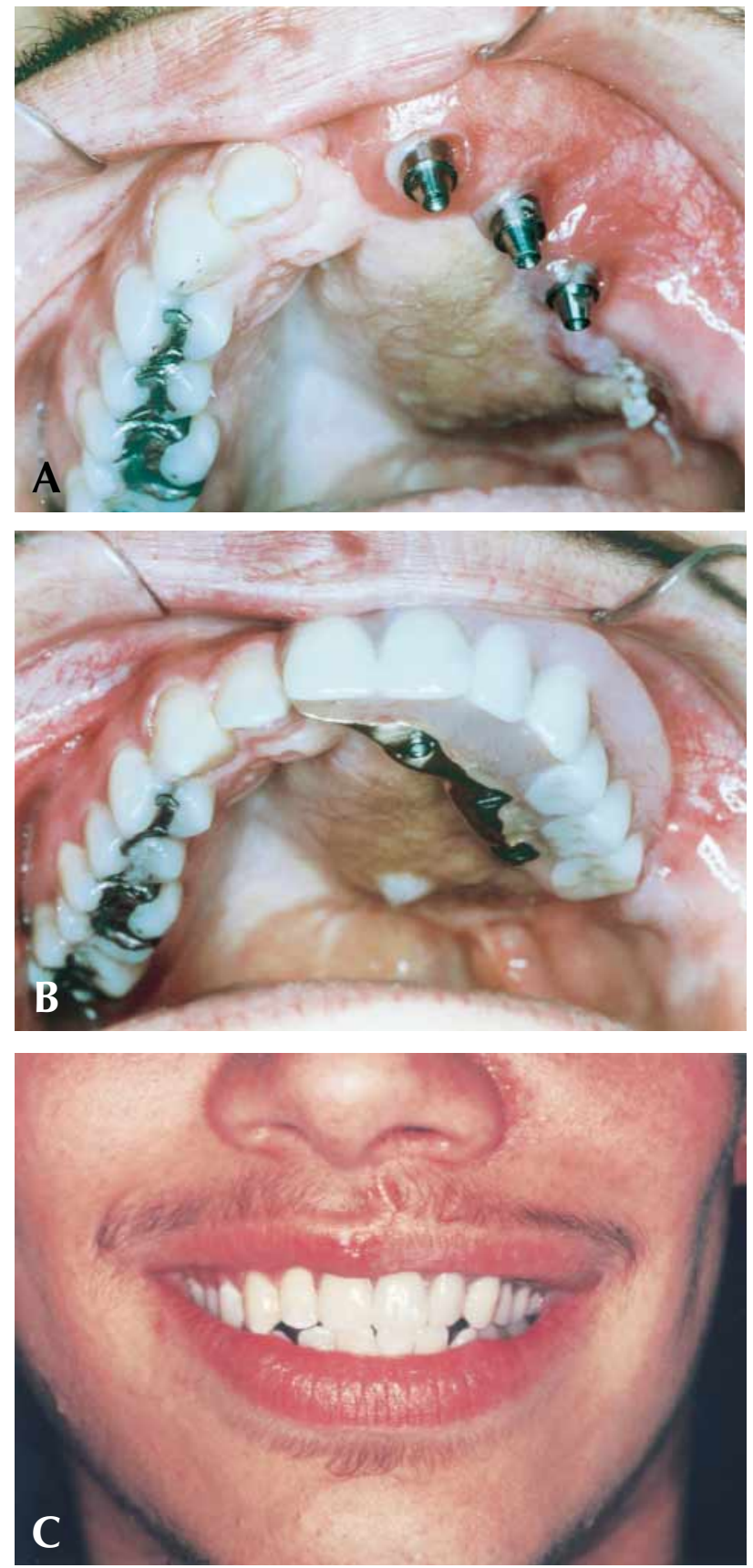

Fig. 2. A, Palatomaxillary reconstruction with scapular osteomyocutaneous free flap and implants. B, Implantretained fixed-hybrid prosthesis restored occlusion over stable maxillary base. C, Lip and cheek support provided by prosthesis for symmetry of mouth.

can provide the restoration of a stable palatal base (Fig. 2).

The biomechanical principles relevant to prosthetic rehabilitation of maxillectomy defects should be utilized if surgical reconstruction is anticipated. Indications for the use of fasciocutaneous flaps and VBCFFs can be derived from the application of these biomechanical 


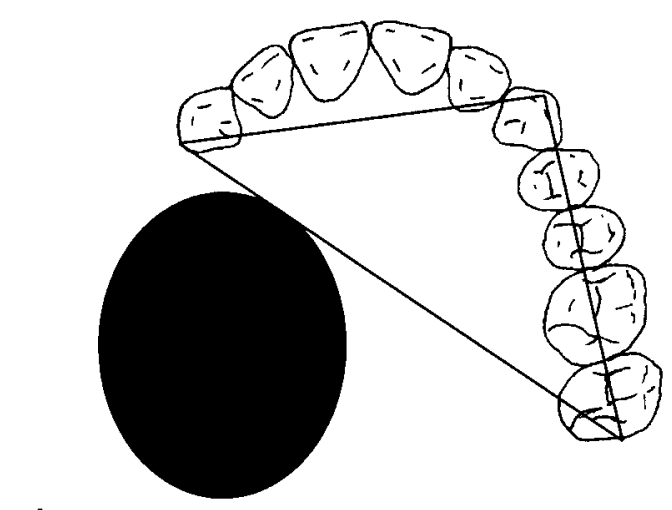

A

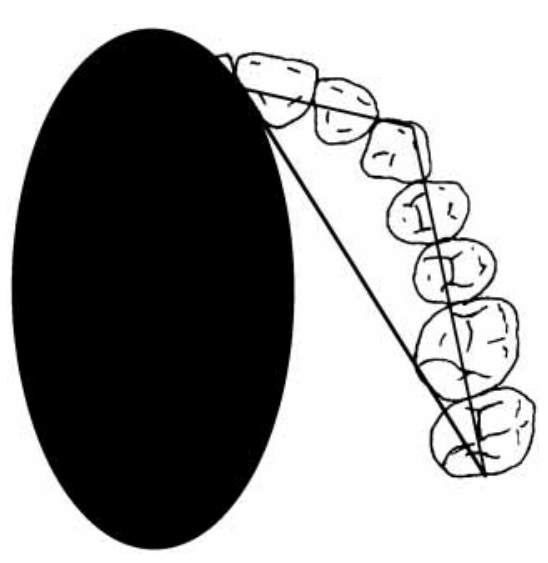

Fig. 3. A, Triangle formed by fulcrum line of framework and tooth farthest from defect. Framework with complete palate major connector and direct retainers on teeth away from fulcrum line will resist destabilizing forces and contribute to stabilization of obturator. B, Supporting tissues diminish as defect enlarges, affecting prosthetic stability. Dental arch shortens, remaining palate decreases, and superior root form of canine, as terminal abutment, is replaced with that of incisor. All factors can contribute to instability of obturator.

principles and from the anatomy of the remaining dental arch and palate.

\section{FUNCTIONAL ANATOMY FOR SURGICAL RECONSTRUCTION}

The maxilla comprises the paired structures of the right and left maxillae. ${ }^{17}$ The body of each maxilla is hollow and shaped like a pyramid, with the base situated medially and adjacent to the nasal cavity. When the anatomy relevant to palatomaxillary reconstruction is considered, the maxilla can be conveniently divided into supportive buttresses and processes. The former constitute the foundation essential for resisting the forces of mastication, and the latter are responsible for the form of the palate and midface. Ideally, the surgical reconstruction of the palatomaxillary defect should address both anatomic units.

The anatomic complexity of the maxilla is related to its 3-dimensional construct, a lattice-like structure that is supported by 3 separate buttresses. These buttresses, which form as an adaptation to the vertical forces of mastication, are the nasomaxillary, zygomaticomaxillary, and pterygomaxillary buttresses. The integrity of these structures is essential to providing a stable occlusal surface for the mandible. Furthermore, they allow for an even distribution of forces across the skull base.

There are 4 processes related to the maxilla: zygomatic, alveolar, palatine, and frontal. The zygomatic and alveolar processes play a key role in the form of the midface. The zygomatic process is responsible for symmetry and projection of the malar eminence. Reconstruction of the maxilla may require reconstitution of the hard palate, lateral nasal wall, alveolus, and anterior face of the maxilla. In some situations, reconstruction of the zygoma and orbital floor is required. These structures are responsible for both cosmetic and functional characteristics of the midface. Reconstitution of the buttress system and attention to the processes ensure a stable base for occlusion, which is essential to optimal functional and esthetic rehabilitation.

\section{PROSTHETIC PROGNOSIS AND SURGICAL RECONSTRUCTION}

Every effort should be made to re-establish a favorable distribution of force to achieve stabilization of an obturator prosthesis during mastication and function. ${ }^{18-20}$ The distribution of force derived from the metal framework and obturator bulb emulates the stable base that the native maxilla can provide for function. The engagement of structures within the defect diminishes the counterproductive lever forces placed on the obturator; contributes to the support, stability, and retention of the prosthesis; and may increase its success. The size and location of the defect, remaining dentition, and supporting surface area of the remaining palate primarily determine the stability and retention of an obturator. As the size of the maxillary defect increases and the remaining dentition and palatal supporting area decrease, the ability to stabilize at the fulcrum line of an obturator framework diminishes. The terminal abutment teeth of the remaining arch determine the fulcrum line. When 2 lines are drawn from the fulcrum line to the canine away from the defect, a stable triangle is established (Fig. 3, A). The hypotenuse of the triangle represents the fulcrum line. When the defect enlarges and the remaining 
palate and dental arch decrease, the area within the triangle diminishes, as does the stability of the prosthesis (Fig. 3, B). The rotation of the obturator framework around the fulcrum line occurs as a result of the differences in quality and quantity of supporting tissues on either side of the fulcrum line. A number of postoperative techniques can be incorporated in the design of the prosthesis to help minimize the cantilever forces and thereby improve stabilization.

Direct retainers of the framework that clasp the remaining dentition are essential to neutralizing adverse cantilever forces. The root form and periodontal condition of abutment teeth are paramount. The condition of the tooth closest to the defect plays an essential role in determining prosthetic prognosis because it will bear the greatest stress. ${ }^{21}$ Hence, adequate root form of this terminal abutment is important. This is exemplified by the large discrepancy between the superior root form and surface area of the canine tooth compared with that of the lateral incisor. Defects that require resection of the canine tooth and a portion of the premaxilla can result in a terminal abutment tooth with an inferior root form adjacent to a large defect. Large defects leave less dentition available to clasp. The diminished palatal supporting surface area results in greater cantilever forces around the fulcrum line of an obturator framework, and as a result, the prosthesis tends to tip toward the defect. Techniques to neutralize these counterproductive forces become more challenging with extensive palatal defects, and the prognosis for functional recovery accordingly becomes less favorable. ${ }^{22}$ Adverse effects are more likely to occur, and the patient's quality of life may suffer.

Acquired defects of the maxilla also can be surgically reconstructed. A comprehensive treatment approach can provide a more conventional setting for prosthodontic rehabilitation. When both are successful, function and cosmesis can be restored. This has been demonstrated with microvascular free flap reconstruction of the mandible. ${ }^{23-27}$ Predictable implant placement in VBCFFs also allows for successful prosthetic rehabilitation. ${ }^{28-32}$ Unlike mandible reconstruction, in which VBCFFs are necessary for discontinuity defects, limited defects of the palate can be reconstructed with a soft tissue free flap, provided that the native maxilla maintains the stable base necessary for function. Surgical closure of a large maxillary defect, however, does require a VBCFF to re-establish a stable base for function. The use of vascularized bone can allow for predictable osseointegration of dental implants for an implant-retained prosthesis. This approach restores the stable base for occlusion and function. Consideration of the tongue in palatomaxillary reconstruction is limited to providing a sufficient dimension of functional space above the tongue's dorsal surface.

\section{PROPOSED CLASSIFICATION SYSTEM}

Defect classification systems enable surgeons and prosthodontists to use the characteristics of a particular defect to establish a functional prognosis. Since the publication of Ohngren's classification system for maxillectomy defects in 1933,33 a number of oncologically oriented classification schemes have served to describe the anatomic boundaries of the maxillectomy defect. ${ }^{34-36}$ Few classification systems, however, address the issues specifically related to reconstruction of the palatomaxillary defect.6,37

Aramany's classification system, ${ }^{6,7}$ reported in 1978, is referenced frequently in the prosthodontic literature. As mentioned previously, it addresses removable partial framework design and prosthetic rehabilitation of the partially edentulous maxillectomy patient in 6 categories. Spiro et $\mathrm{al}^{36}$ proposed a relatively simple classification system in 1997 that focuses on infrastructure defects; however, it does not specifically address the involvement of adjacent structures such as the orbit and zygoma. It was suggested that defects be termed as "limited" or "subtotal" on the basis of the number of maxillary "walls" involved in the resection.

Realizing that the technique employed for optimal maxillary reconstruction was largely dependent on the nature and extent of the defect, Davison et $\mathrm{al}^{37}$ proposed a reconstruction algorithm based on a review of 108 patient treatments involving prosthetic obturation, nonvascularized bone grafts, local flaps, regional flaps, and microvascular free tissue transfer. He divided patients into the 2 broad categories of "complete" and "partial" maxillectomy defects. Davison proposed a wide range of reconstructive techniques, but the lack of a specific defect-oriented classification system outlining the remaining portion of the hard palate, dentition, orbit, and zygoma makes such an algorithm difficult to apply as a reconstructive guide.

Brown ${ }^{13}$ was the first to discuss a multidisciplinary (surgical and prosthodontic) approach to palatomaxillary reconstruction. His more useful defect classification scheme is based on both the vertical and horizontal dimensions of a defect. The vertical component of the defect ranges from minor resections with no oro-antral fistula to radical maxillectomy defects with orbital exenteration. The horizontal component ranges from a small hard palate defect without involvement of dentition to a total palatectomy defect. According to Brown's scheme, the classification of the horizontal dimension of the defect is based on the remaining palatal surface. Although the residual palatal surface plays an important role in determining the best form of reconstruction for the edentulous patient, the dentate patient can rely not only on the remaining palate but also on the remaining dentition for retention. Furthermore, the state of the orbital floor and zygoma, which play an important role 

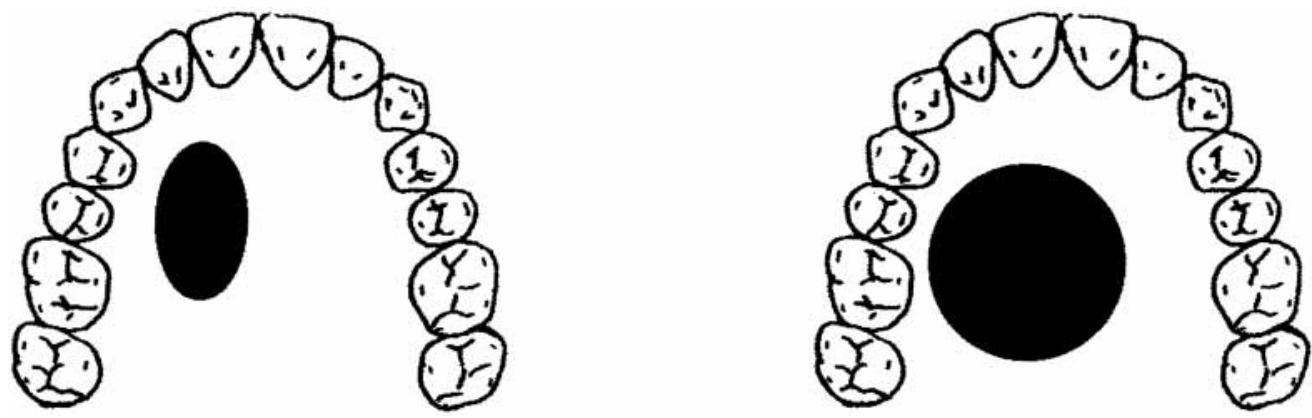

Fig. 4. Class la defects involve any portion of hard palate but not tooth-bearing maxillary alveolus.

in both the function and cosmetic results of the midface, are not specifically addressed in Brown's system. Defects involving either of these areas are poorly managed by prosthetic rehabilitation and, as a result, often require hard tissue reconstruction.

To assess the functional outcome and patient satisfaction that surgical reconstruction can provide, a classification system of defects based on a selected patient population at Mount Sinai Medical Center (New York, N.Y.) was established. A retrospective review of 47 consecutive palatomaxillary restorations at a tertiary referral center was performed. Patient treatments were entered into the study if they involved resection of the hard palate or palatectomy in combination with a partial or total maxillectomy. All of the defects reviewed were rehabilitated with a tissue-borne obturator, a local palatal island flap, a fasciocutaneous free flap, or a vascularized bone containing free flap. All patients had been offered preoperative consultations with a head and neck microvascular surgeon, an oral maxillofacial surgeon, and a maxillofacial prosthodontist. Design considerations for surgical reconstruction and prosthodontic rehabilitation focused on 4 objectives: (1) closure of the oral cavity, (2) provision of a stable base for the restoration of function, (3) restoration of midface symmetry, and (4) support of orbital structures.

Twenty patients had been rehabilitated with a tissue-borne obturator and 27 patients with surgical closure of the oral cavity. Of the surgical closure patients, 5 were reconstructed with local palatal island flaps, 4 with fasciocutaneous free flaps, and 18 with bone-containing free flaps. With the use of the biomechanical principles outlined above, palatomaxillary defects were divided into 3 major classes and 2 subclasses. Eight different defects of the hard palate and maxilla were characterized within this classification system. The size and location of the defect, remaining dentition, and palate influenced the design of the microvascular free flap and prosthodontic restoration.
Maxillectomy defects involving the floor of the orbit and/or zygoma also played a role in the donor site selection and design of the microvascular free flap.

\section{Class Ia}

Defects that involved the hard palate but not the tooth-bearing alveolus were categorized as Class Ia (Fig. 4). These defects could be rehabilitated with an obturator, a local advancement flap, or a fasciocutaneous free flap. In general, prostheses created for prosthetic obturation were stable and well tolerated. However, while prosthetic rehabilitation offered the advantage of immediate orodental rehabilitation without the need for further surgery, some patients found that the maintenance of prosthetic closure was inconvenient. Local island flaps offered a simple method of primary reconstruction of palatal defects involving less than one third of the hard palate. Palatal flap reconstruction also obviated the need for a dental prosthesis. If a palatal defect was not amenable to a local advancement flap reconstruction because the patient was previously irradiated or the defect was too large, the defect was reconstructed with a fasciocutaneous free flap.

\section{Class Ib}

Defects that involved any portion of the maxillary alveolus and dentition posterior to the canines or that involved the premaxilla were categorized as Class $\mathrm{Ib}$ (Fig. 5). These defects involved a small portion of the dental arch; the anterior sextant and a unilateral posterior quadrant of teeth remained intact. As a result, the theoretic cantilever forces over the defect were minimized. It was projected that the movement of the obturator around the fulcrum line could be stabilized. Factors that contributed to stability of the framework of Class Ib patients included the superior root morphology of the canine approximating abutment and the considerable arch length provided by a sound anterior 

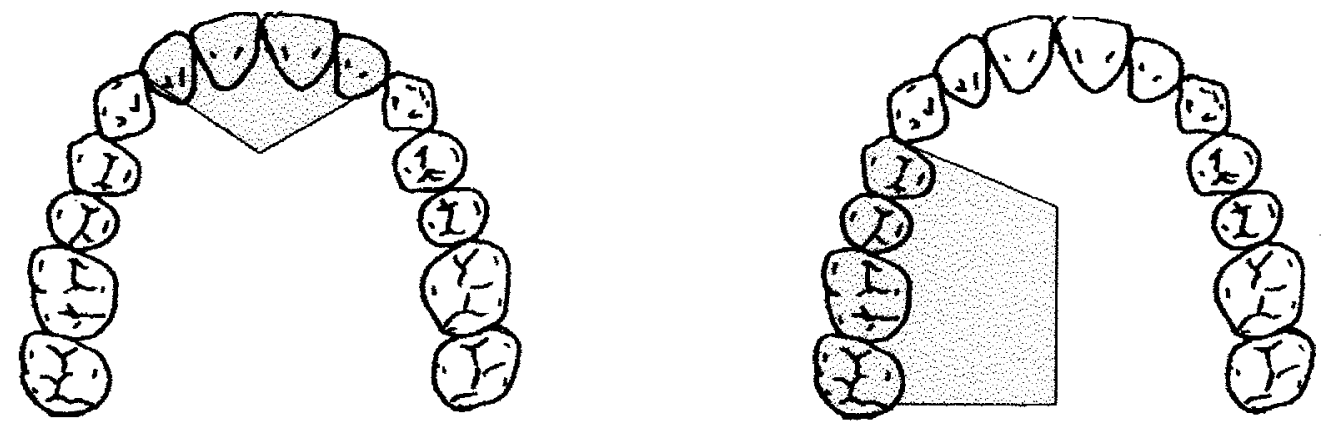

Fig. 5. Class Ib defects involve premaxilla or any portion of maxillary alveolus and dentition posterior to canines.
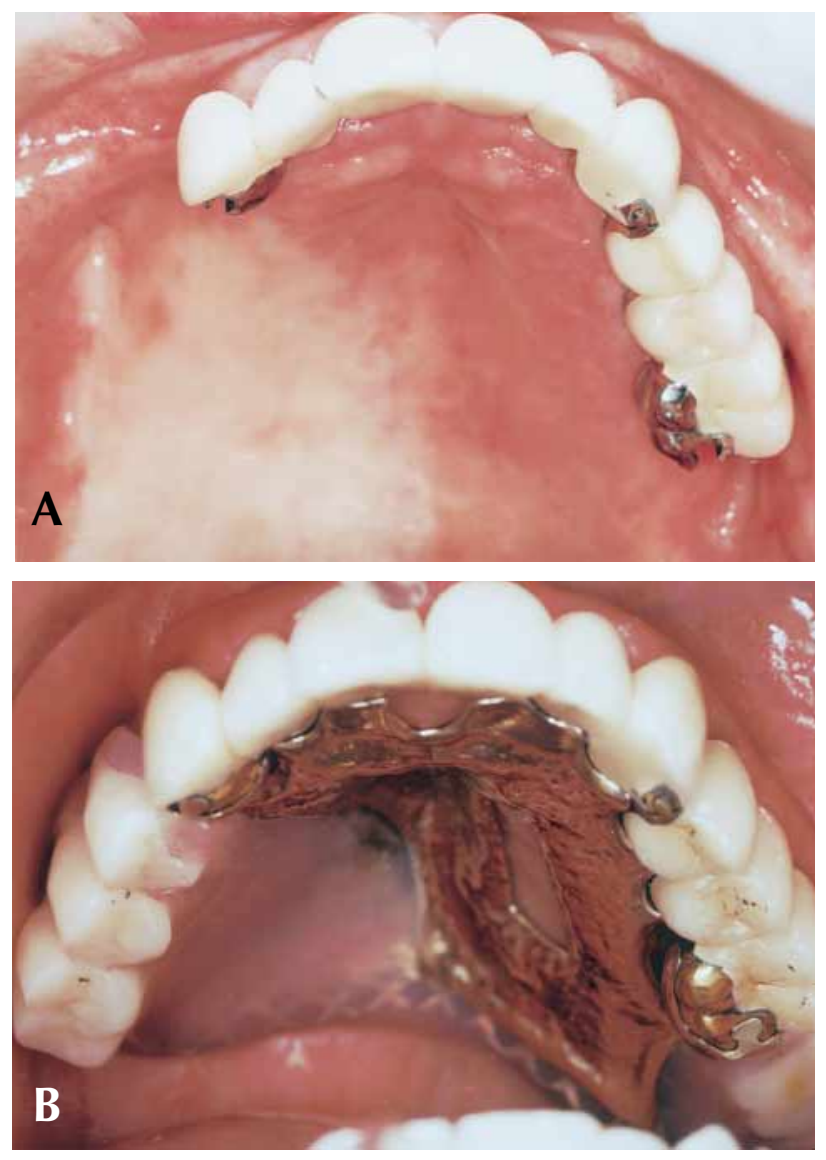

sextant and unilateral posterior quadrant. The ability to clasp teeth perpendicular to the fulcrum line of the framework and the support afforded by the remaining palate further stabilized the framework of the prosthesis and improved the prosthetic prognosis.

If surgical reconstruction was planned, a soft tissue flap was indicated without osseous reconstruction because the remaining dentition and palate were considered able to support occlusal contacts over the reconstruction with a removable partial denture. With removable prosthodontic treatment, support is not

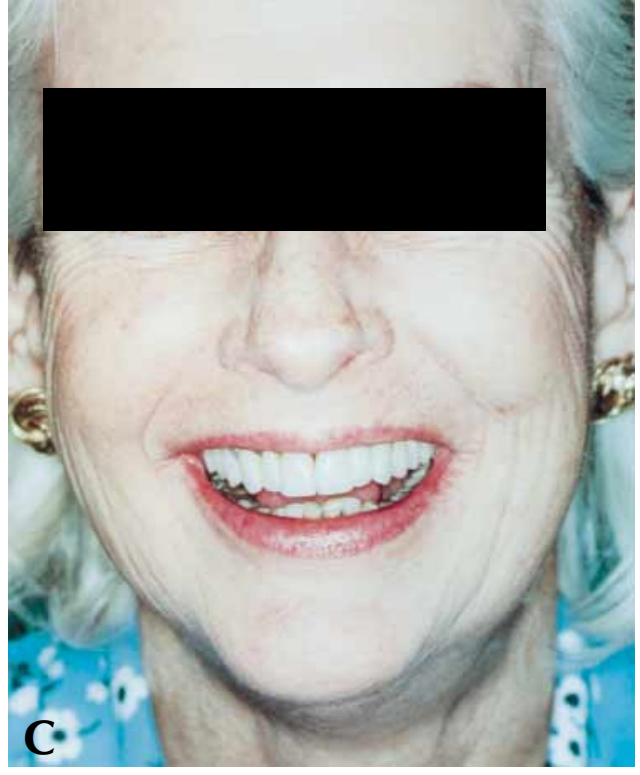

Fig. 6. A, Class Ib defect 2 years after immediate reconstruction with radial forearm fasciocutaneous free flap. B, Prosthodontic rehabilitation with connected crowns and semiprecision removable partial denture. Remaining dentition was evaluated at presurgical consultation to determine whether it could stabilize removable prosthesis and support occlusal contacts over reconstruction. C, Surgical reconstruction and prosthodontic rehabilitation provided functional and esthetic results.

provided by the fasciocutaneous free flap but derived from the remaining dentition and palate. A radial forearm fasciocutaneous free flap worked well for closure of the oral cavity because of ample soft tissue of the donor site and low donor site morbidity relative to those of other sites (Fig. 6).

In the edentulous patient, the bone of the remaining maxillary alveolus had to be sufficient to accommodate osseointegrated implants. Forgoing osseointegration of implants in the remaining natural maxilla and surgical closure of the defect with a fascio- 

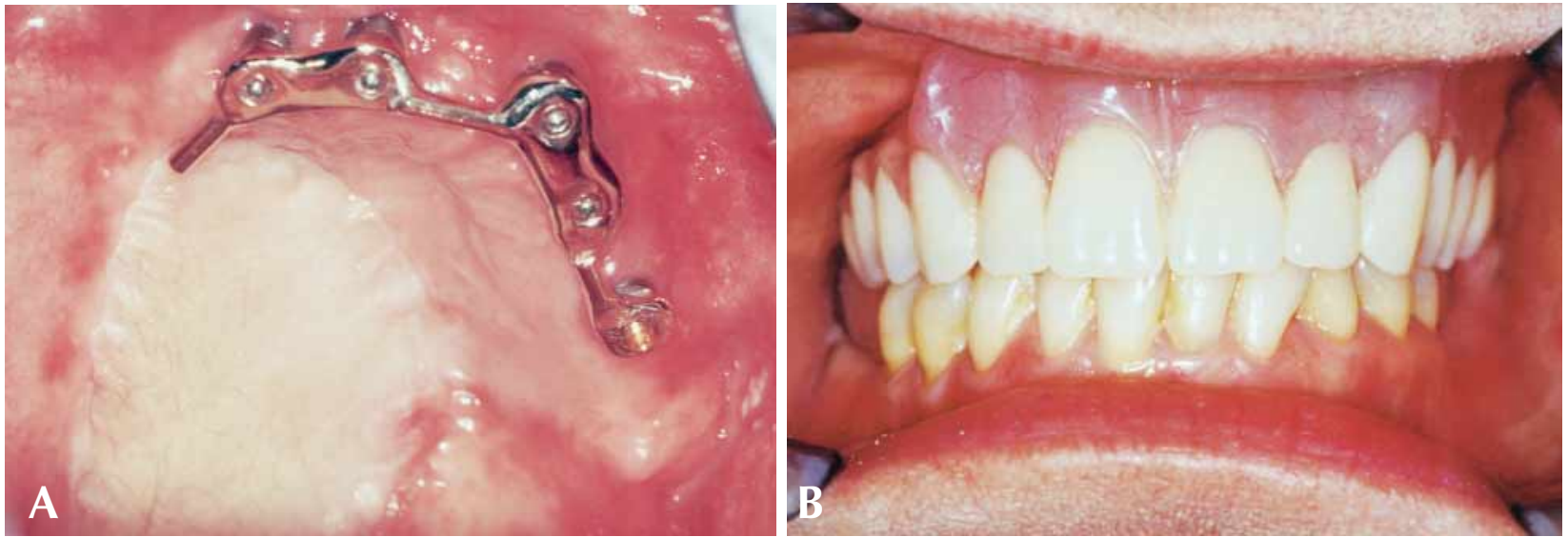

Fig. 7. A, Immediate reconstruction of edentulous Class Ib defect with radial forearm fasciocutaneous free flap. Osseointegrated implants were placed at time of surgical reconstruction in native alveolus. B, Gold bar and clips provided stabilization for complete overdenture. Failure to consider osseointegration at time of free flap reconstruction in edentulous arch may make prosthodontic rehabilitation difficult.
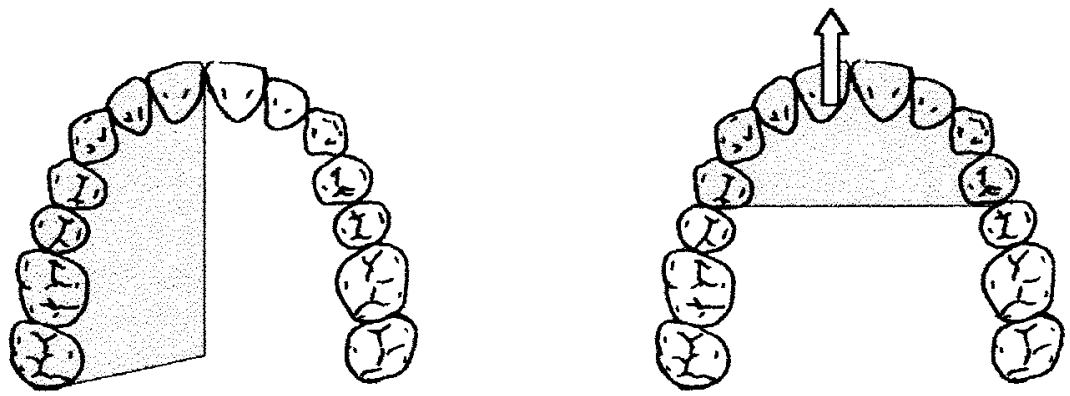

Fig. 8. Class II defects involve any portion of hard palate and tooth-bearing maxillary alveolus and only one canine. Anterior margin of defect lies within premaxilla. This class includes transverse palatectomy defects that involve less than $50 \%$ of hard palate.

cutaneous free flap can result in a difficult or impossible situation for prosthetic rehabilitation. This is due to the inability to extend the obturator bulb into the defect and engage anatomic undercuts for stability and retention (Fig. 7).

\section{Class II}

Defects that involved any portion of the toothbearing maxillary alveolus but included only 1 canine were categorized as Class II. The anterior margin of these defects was within the premaxilla. Also included in this class were anterior transverse palatectomy defects that involved less than one half of the palatal surface (Fig. 8).

The total maxillectomy comprised the majority of defects in this class. An incisor served as a ter- minal abutment. Prosthetic rehabilitation of Class II defects was less predictable than that of Class I defects. Factors that contributed to instability included fewer teeth for clasping, reduced arch size and form, and a significantly diminished supporting palate. An obturator alone was inadequate to restore cosmesis to the midface if the orbital floor or the zygoma was resected.

Some Class II defects were best reconstructed and rehabilitated by VBCFFs. Vascularized bone offered the ability to re-establish the bony dental arch for the placement of osseointegrated implants. This allowed for the distribution of masticatory forces across an intact maxillary arch and thereby reestablished a favorable biomechanical condition to the maxilla. Furthermore, the VBCFFs permitted the 

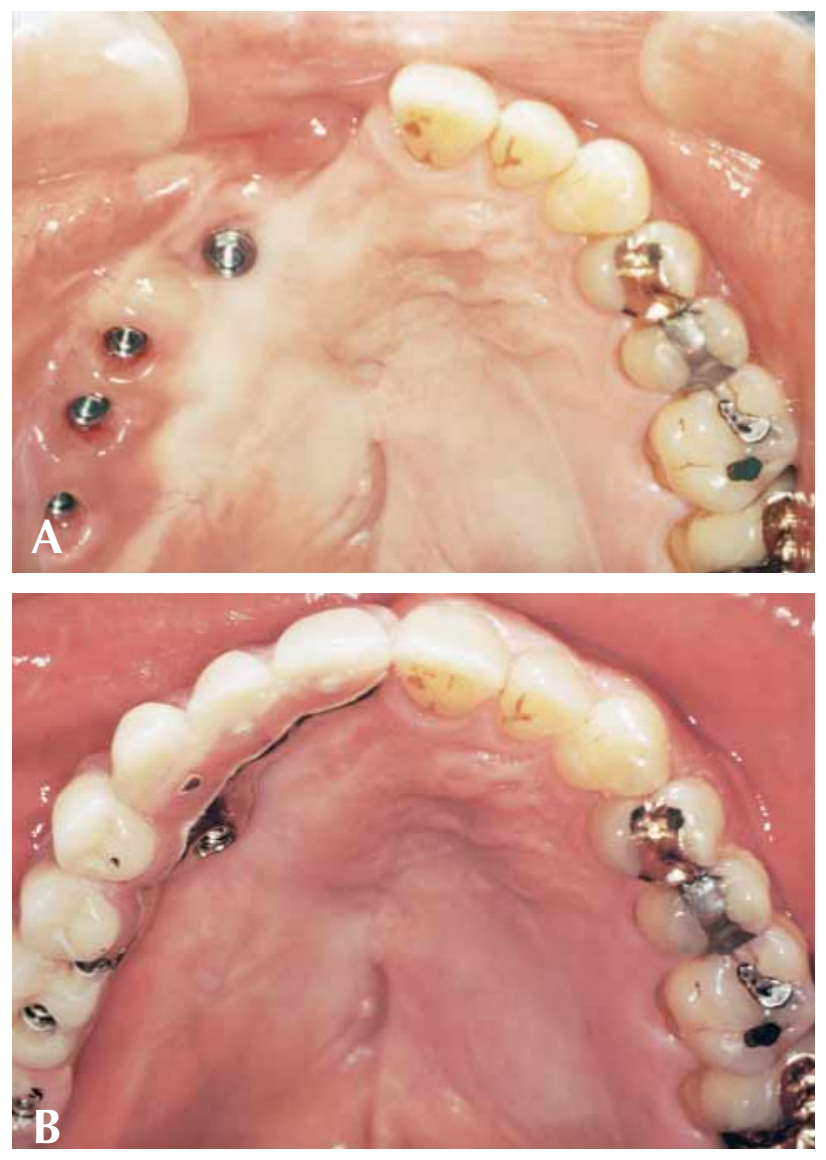

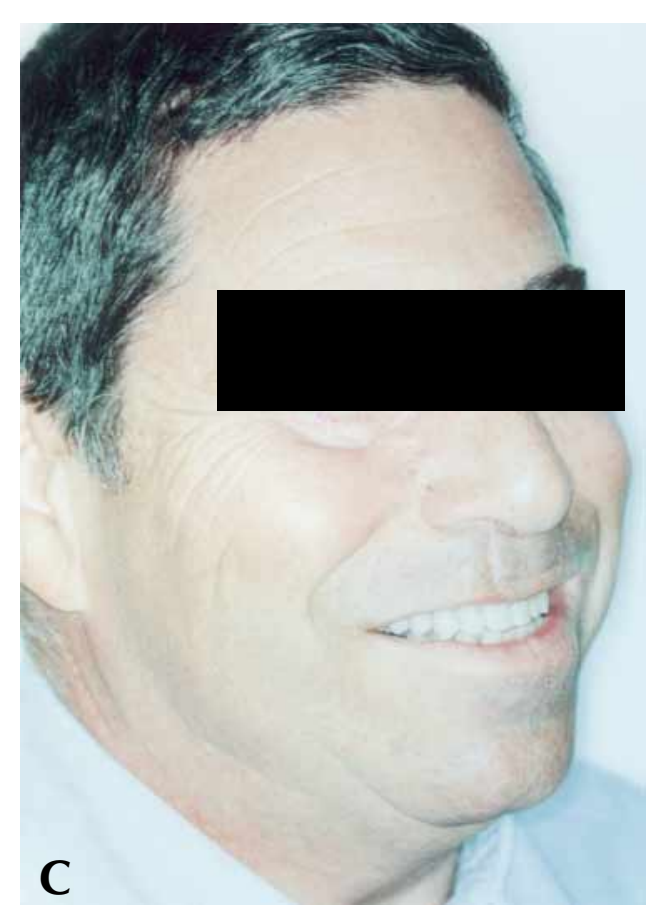

Fig. 9. A, Class Ilfz defect reconstructed with iliac crest-internal oblique muscle osteomyocutaneous free flap. B, Osseointegration in vascularized bone provided support for fixed-hybrid prosthesis. C, Surgical reconstruction of orbital floor and zygoma provided restoration of midface projection that was unattainable with prosthetic rehabilitation alone.
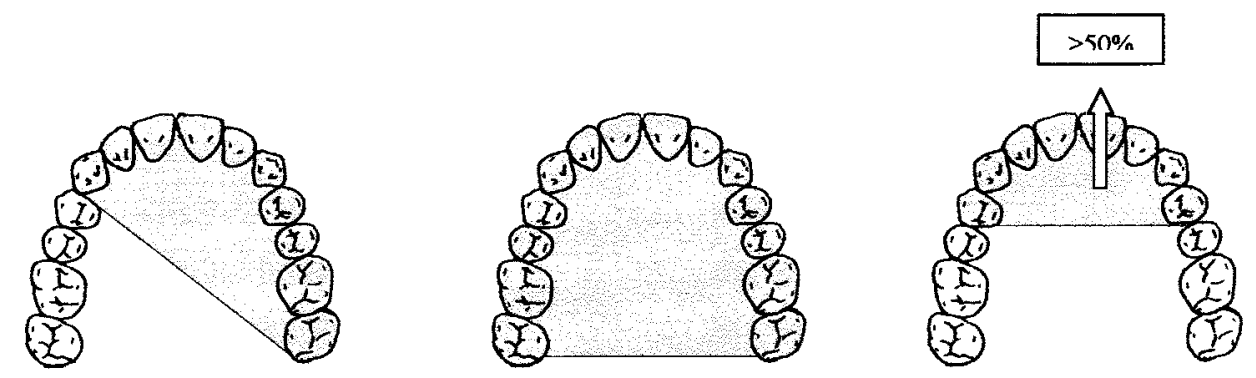

Fig. 10. Class III defects involve any portion of hard palate and tooth-bearing maxillary alveolus, including both canines. This class includes total and transverse palatectomy defects that involve more than $50 \%$ of hard palate.

primary reconstruction of the orbital rim and the prominence of the zygomatic body with autologous tissue (Fig. 9).

\section{Class III}

Defects that involved any portion of the tooth-bearing maxillary alveolus and included both canines, total palatectomy defects, and anterior transverse palatectomy that involved more than half of the palatal surface were categorized as Class III (Fig. 10). These defects left little or no residual palate or dentition for the secure retention of an obturator, which led to a poor prosthetic prognosis. Class III defects were best restored with a VBCFF. Although soft tissue reconstruction of a Class III defect served to effectively partition the oral cavity from the nasal cavities and maxillary sinuses, orodental rehabilitation was severely compromised. Bone-containing free flaps, however, served to separate the oral and nasal cavities while providing vascularized bone capable of retaining implants. Palatal reconstruction with bone provided a stable base to oppose the restored mandibular arch (Fig. 11). 

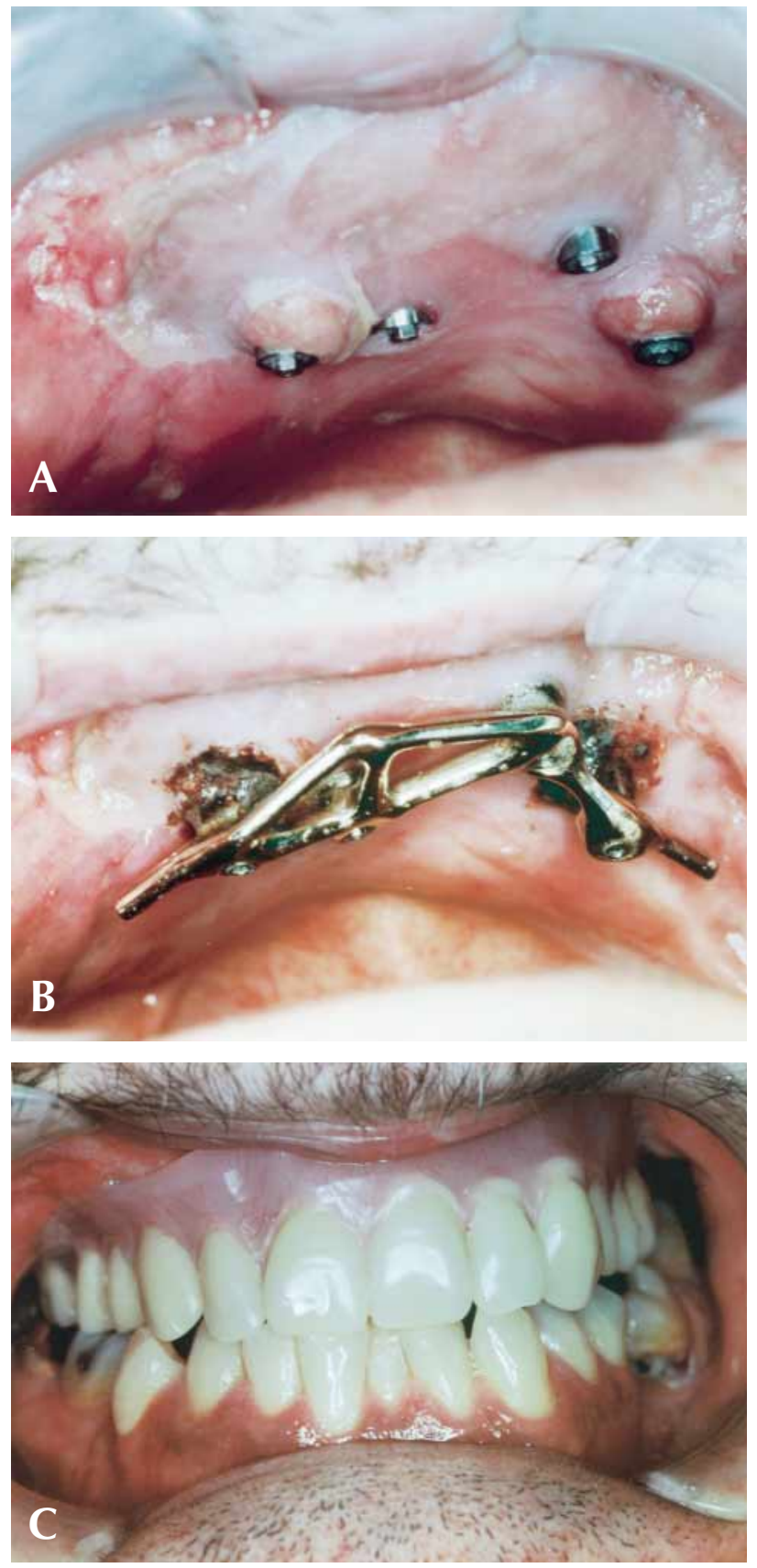

Fig. 11. A, Defect was reconstructed with scapular osteomyocutaneous free flap. Four implants were placed in greatest dimension of scapula. B, Gold bar was designed for implant-retained complete overdenture. C, Occlusion was restored over stable base provided by vascularized bonecontaining free flap.

\section{Subclasses $\mathrm{f}$ and $\mathrm{z}$}

Defects that involved the inferior orbital rim were categorized as subclass $f$, whereas defects that involved the body of the zygoma were categorized as subclass z (Fig. 12). Extensive palatomaxillary
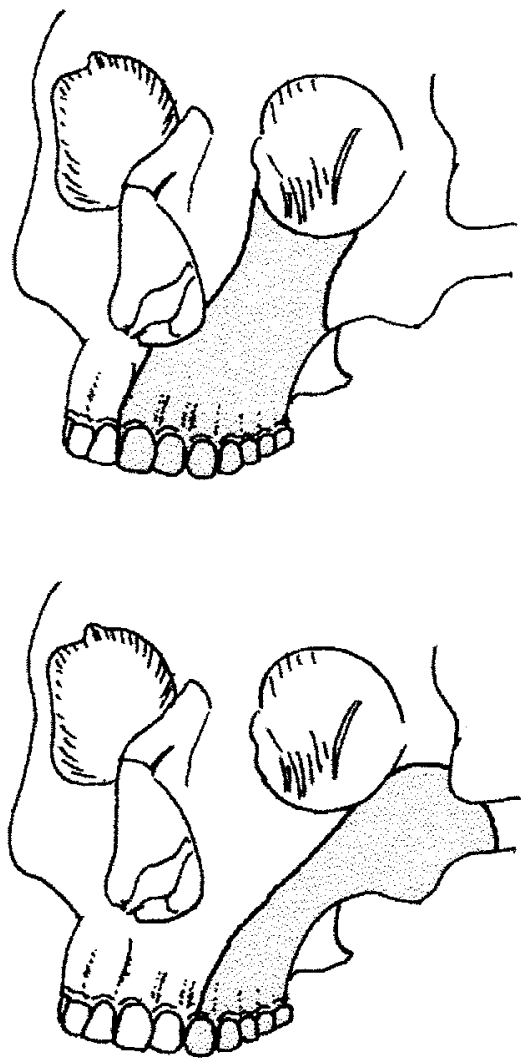

Fig. 12. A, Subclass $f$ defects involve orbital floor. B, Subclass $z$ defects involve any portion of zygomatic body.

defects commonly involve a vertical component of the maxilla. The creation of subclasses related to the status of the orbital floor and the zygomatic body was essential to provide an accurate description of the palatomaxillary defect. As stated earlier, the orbital floor and zygomatic body play both functional and cosmetic roles. Ablation of the vertical maxilla commonly results in a significant disruption of the midface and orbit and has a profound impact on function. Enophthalmoses and diplopia can occur if the orbital contents are not supported. In addition, these patients may suffer from a cosmetic deficit (caused by the loss of midface symmetry and projection) that is almost impossible to restore with an obturator.

In our experience, VBCFFs served to restore bone to the load-bearing palate by re-establishing the vertical buttress system and restoring bone to the orbital rim and zygomatic body. The combination defect after maxillectomy and orbital exenteration could be surgically reconstructed with a VBCFF. Closure of the oral cavity was attained, and reconstruction of the orbital rim with vascularized bone permitted the placement of 

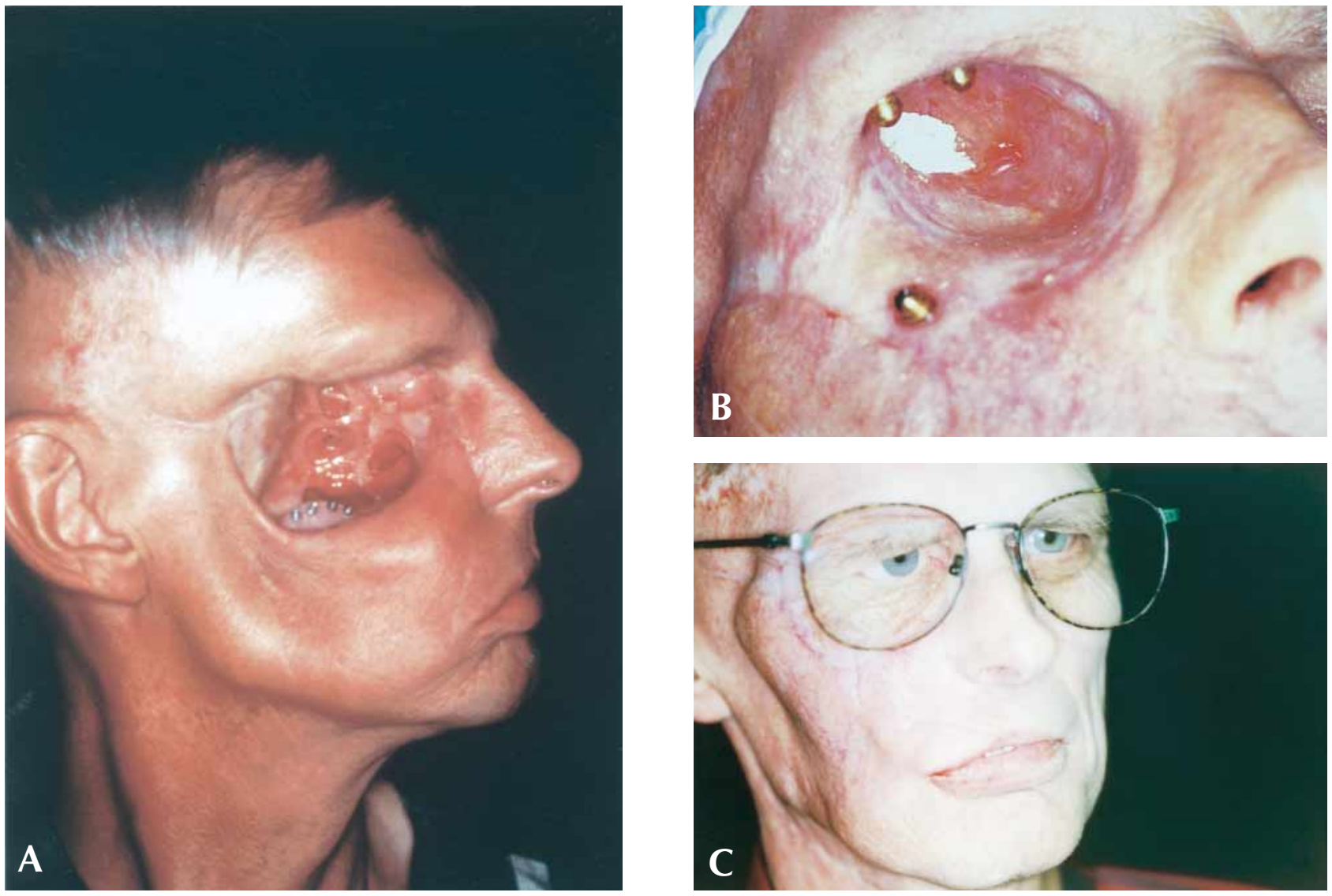

Fig. 13. A, After hyberbaric oxygen protocol, patient presented with osteoradionecrosis of maxillectomy-orbitectomy defect (Class IIfz). B, Free flap donor site and design satisfied needs of defect. Surgical reconstruction involved orbital floor, zygoma, midface, and palate with iliac crest-internal oblique muscle osteomyocutaneous free flap. C, Osseointegrated implants in vascularized bone and superior orbital rim provided magnet retention for orbital prosthesis that restored midface projection and symmetry.
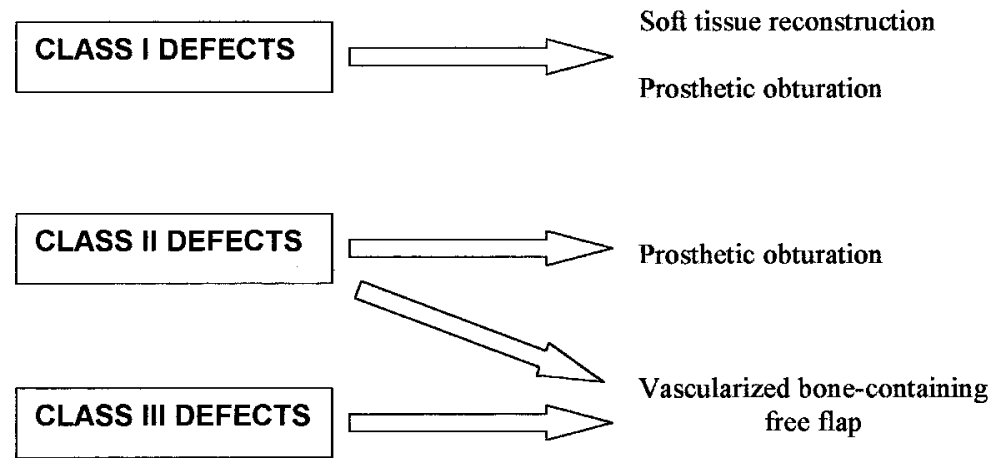

Fig. 14. Algorithm for palatomaxillary reconstruction.

osseointegrated implants for the retention of an orbital prosthesis (Fig. 13).

\section{DISCUSSION}

The palatomaxillary classification system presented here was based on clinical experience at Mount
Sinai Medical Center, which was used to establish an algorithm for functional reconstruction, midface restoration, and orodental rehabilitation (Fig. 14). This system may provide a methodology to enroll larger patient numbers from multiple institutions to study various methods of rehabilitation. 
The defect-oriented approach of the proposed system is intended to facilitate and coordinate treatment planning among surgeons and prosthodontists. The surgical approach should facilitate functional rehabilitation. New surgical reconstruction techniques may or may not provide a more conventional setting for prosthodontic rehabilitation. Comprehensive treatment planning is necessary to meet multidisciplinary objectives for patients with complex reconstructive and rehabilitative needs.

Our experience with palatomaxillary reconstruction and prosthodontic rehabilitation highlights the range of options available to the reconstructive surgeon and the necessity to combine these techniques to achieve a functional result. Class I palatomaxillary defects can be rehabilitated with a prosthetic obturator or soft tissue to achieve optimal orodental restoration. Soft tissue reconstruction can be achieved with either a local advancement flap or a fasciocutaneous free flap. Defects that involve an ipsilateral single canine (Class II) can be rehabilitated with an obturator prosthesis or a VBCFF. While pure Class II defects are amenable to prosthetic obturation, Class IIfz defects are best managed with a VBCFF, which re-establishes the alveolar arch and provides a stable base for function and implant prosthodontics. The VBCFF also offers the opportunity to reconstruct the orbital rim/floor as well as the zygomatic defect.

Similar biomechanical principles can be applied to the transverse palatectomy defect. Defects that involve less than $50 \%$ of the transverse palate can be rehabilitated effectively with a prosthetic obturator. A maxillectomy defect in a vertical orientation has a more profound effect on prosthetic stability than an anterior palatal defect. Loss of the anterior nasal spine can profoundly affect facial cosmesis, and prosthetic treatment alone may not be able to restore the midface projection to its original dimensions.

Reconstruction of the anterior maxillary arch with a VBCFF is another option for these patients. Reconstruction of the dental arch and inferior piriform aperture with vascularized bone may allow for the placement of osseointegrated implants, a favorable distribution of functional forces, and a surgical restoration of normal midface projection. In addition, the use of a VBCFF, osseointegrated implants, and a nasal prosthesis may be an effective approach to restoring a combined defect involving rhinectomy and maxillectomy. A nasal prosthesis may provide a superior alternative to surgical reconstruction for large rhinectomy defects because of the near normal resemblance of cartilaginous structures such as the alar rim and tip of the nose. These structures are difficult to reconstruct with connective tissue and skin provided by a free flap. Microvascular free flap surgi- cal reconstruction of the nose may be better suited for subtotal rhinectomy-maxillectomy defects.

Similarly, Class III defects require vascularized bone for optimal orodental rehabilitation. Prosthetic obturation of these defects often is associated with a poor seal resulting from the instability of the prosthesis. Compromised function may lead to poor quality of life. In our experience, these defects are best managed with VBCFFs and osseointegration.

Several factors that must be considered when surgical reconstruction is planned are not addressed by the proposed classification system. The extent of tumor invasion into adjacent structures and the tumor's biologic behavior may affect the options related to restorative care. Similarly, a range of patient factors may influence the reconstructive approach; these include age, comorbidities, prior surgery and/or radiation, patient motivation, financial considerations, and prosthodontic prognosis.

\section{SUMMARY}

The classification system presented in this article may facilitate further study of the surgical reconstruction and prosthodontic rehabilitation of maxillectomy defects. Although the system does not address all factors related to the restorative decision-making process, it is intended as a guide and algorithm for reconstruction of the palatomaxillary defect.

\section{REFERENCES}

1. Minsley GE, Warren DW, Hinton V. Physiologic responses to maxillary resection and subsequent obturation. J Prosthet Dent 1987;57:338-44.

2. Watson RM, Gray BJ. Assessing effective obturation. J Prosthet Dent 1985; 54:88-93.

3. Shipman B. Evaluation of occlusal force in patients with obturator defects. J Prosthet Dent 1987;57:81-4.

4. Desjardins RP. Obturator prosthesis design for acquired maxillary defects. J Prosthet Dent 1978;39:424-35.

5. Beumer J, Curtis T, Marunick M. Maxillofacial rehabilitation: prosthodontic and surgical considerations. St. Louis: Ishiyaku EuroAmaerica; 1996. p. 233-7.

6. Aramany MA. Basic principles of obturator design for partially edentulous patients. Part I: classification. J Prosthet Dent 1978;40:554-7.

7. Aramany MA. Basic principles of obturator design for partially edentulous patients. Part II: design principles. J Prosthet Dent 1978;40:656-62.

8. King GE, Gay WD. Application of various removable partial denture design concepts to a maxillary obturator prosthesis. J Prosthet Dent 1979;41:316-8

9. Parr GR, Tharp GE, Rahn AO. Prosthodontic principles in the framework design of maxillary obturator prostheses. J Prosthet Dent 1989;62:205-12.

10. Martin JW, King GE. Framework retention for maxillary obturator prostheses. J Prosthet Dent 1984;51:669-72.

11. Funk GF, Laurenzo JF, Valentino J, McCulloch TM, Frodel JL, Hoffman HT. Free-tissue transfer reconstruction of midfacial and cranio-orbito-facial defects. Arch Otolaryngol Head Neck Surg 1995;121:293-303.

12. Muzaffar AR, Adams WP Jr, Hartog JM, Rohrich RJ, Byrd HS. Maxillary reconstruction: functional and aesthetic considerations. Plast Reconstr Surg 1999;104:2172-83.

13. Brown JS. Deep circumflex iliac artery free flap with internal oblique muscle as a new method of immediate reconstruction of maxillectomy defect. Head Neck 1996;18:412-21.

14. Funk GF. Scapular and parascapular free flaps. Facial Plast Surg $1996 ; 12: 57-63$ 
15. Futran ND, Haller JR. Considerations for free-flap reconstruction of the hard palate. Arch Otolaryngol Head Neck Surg 1999;125:665-9.

16. Chen HC, Ganos DL, Coessens BC, Kyutoku S, Noordhoff MS. Free forearm flap for closure of difficult oronasal fistulas in cleft palate patients. Plast Reconstr Surg 1992;90:757-62.

17. Larabee WF, Makielski KH. Surgical anatomy of the face. New York: Raven Press; 1993. p. 31-2.

18. Brown KE. Peripheral consideration in improving obturator retention. J Prosthet Dent 1968;20:176-81.

19. Myers RE, Mitchell DL. A photoelastic study of stress induced by framework design in a maxillary resection. J Prosthet Dent 1989;61:590-4.

20. Schwartzman B, Caputo A, Beumer J. Occlusal force transfer by removable partial denture designs for a radical maxillectomy. J Prosthet Dent 1985:54:397-403.

21. Fiebirger GE, Rahn AO, Lundquist DO, Morse PK. Movement of abutments by removable partial denture frameworks with a hemimaxillectomy obturator. J Prosthet Dent 1975;34:555-61.

22. Firtell DN, Grisius RJ. Retention of obturator-removable partial dentures: a comparison of buccal and lingual retention. J Prosthet Dent 1980;43:212-7

23. Urken ML, Vickery C, Weinberg H, Buchbinder D, Lawson W, Biller HF. The internal oblique-iliac crest osteomyocutaneous free flap in oromandibular reconstruction. Report of 20 cases. Arch Otolaryngol Head Neck Surg 1989;115:339-49.

24. Urken ML, Weinberg H, Vickery C, Buchbinder D, Lawson W, Biller HF. Oromandibular reconstruction using microvascular composite free flaps. Report of 71 cases and a new classification scheme for bony, soft-tissue, and neurologic defects. Arch Otolaryngol Head Neck Surg 1991;117:733-44

25. Gullane PJ. Primary mandibular reconstruction: analysis of 64 cases and evaluation of interface radiation dosimetry on bridging plates. Laryngoscope 1991;101(6 Pt 2):1-24.

26. O'Leary MJ, Martin PJ, Hayden RE. The neurocutaneous free fibula flap in mandibular reconstruction. Otolaryngol Clin North Am 1994;27:1081 96.

27. Shpitzer T, Neligan P, Gullane P, Freeman J, Boyd B, Rotstein L, et al. Oromandibular reconstruction with the fibular free flap. Analysis of 50 consecutive flaps. Arch Otolaryngol Head Neck Surg 1997;123:939-44.

28. Branemark PI, Hansson BO, Adell R, Breine U, Lindstrom J, Hallen O, et al. Osseointegrated implants in the treatment of the edentulous jaw. Experience from a 10-year period. Scand J Plast Reconstr Surg Suppl 1977;16:1-132.
29. Adell R, Lekholm U, Rockler B, Branemark PI. A 15-year study of osseointegrated implants in the treatment of the edentulous jaw. Int J Oral Surg 1981;10:387-416.

30. Granstrom G, Bergstrom K, Tjellstrom A, Branemark PI. A detailed analysis of titanium implants lost in irradiated tissues. Int J Oral Maxillofac Implants 1994;9:653-62.

31. Urken ML, Buchbinder D, Weinberg H, Vickery C, Sheiner A, Biller HF. Primary placement of osseointegrated implants in microvascular mandibular reconstruction. Otolaryngol Head Neck Surg 1989;101:5673.

32. Urken M, Buchbinder D, Constantino PD, Sinha U, Okay D, Lawson W, et al. Oromandibular reconstruction using microvascular composite flaps: report of 210 cases. Arch Otolaryngol Head Neck Surg 1998;124:46-55.

33. Ohngren L. Malignant tumors of the maxillo-ethmoidal region. Acta Otolarynogol 1933;19:1476.

34. Earley MJ. Primary maxillary reconstruction after cancer excision. Br J Plast Surg 1989;42:628-37.

35. Sakai S, Fuchihata $\mathrm{H}$, Hamasaki Y. Treatment policy for maxillary sinus carcinoma. Acta Otolaryngol 1976;82:172-81.

36. Spiro RH, Strong EW, Shah JP. Maxillectomy and its classification. Head Neck 1997; 19:309-14.

37. Davison SP, Sherris DA, Meland NB. An algorithm for maxillectomy defect reconstruction. Laryngoscope 1998;108:215-9.

Reprint requests to:

DR DEVIN OKAY

Department of Otolaryngology-Head and Neck Surgery

The Mount Sinai Medical Center

Oral and Maxillofacial Associates, Box 1191

5 EAST 98TH ST, 8TH FLOOR

NEW YORK, NY 10029

FAx: (212)427-4088

E-MAIL: d.okay@mssm.edu

Copyright (C) 2001 by The Editorial Council of The Journal of Prosthetic Dentistry.

$0022-3913 / 2001 / \$ 35.00+0 . \mathbf{1 0} / \mathbf{1} / \mathbf{1 1 9 5 2 4}$

doi:10.1067/mpr.2001.119524

\section{Bound volumes available to subscribers}

Bound volumes of The Journal of Prosthetic Dentistry are available to subscribers (only) for the 2001 issues from the publisher at a cost of $\$ 92.00$ (\$106.00 international) for Vol. 85 (January-June) and Vol. 86 (July-December). Shipping charges are included. Each bound volume contains a subject and author index, and all advertising is removed. Copies are shipped within 30 days after publication of the last issue in the volume. The binding is durable buckram with the journal name, volume number, and year stamped in gold on the spine. Volumes 83 and 84 are also available. Payment must accompany all orders. Contact Mosby, Subscription Customer Service, 6277 Sea Harbor Dr, Orlando, FL 32887, or call 800-654-2452 or 407-345-4000.

Subscriptions must be in force to qualify. Bound volumes are not available in place of a regular Journal subscription. 